\title{
Sistema Único de Saúde: a busca do entendimento de seus princípios e sistemas de valores para profissionais do serviço público
}

\author{
Luira Beth Nunes Alonso, Helga Cristina Hedler e \\ Suely Braga Castilho*
}

\section{Introdução e contexto}

Cultura organizacional compreende um sistema de ideias e convicções compartilhadas pelas pessoas que trabalham em uma organização. São crenças e valores (HoFstede, 1980; 1991), tradições, normas, papéis e comportamentos estabelecidos pela organização que podem ser objeto de gestão do conhecimento e de competências. A cultura de uma organização está presente nos sistemas e processos de trabalho construídos ao longo do tempo e afeta o funcionamento de sua totalidade. Quando uma organização passa por transformações, em especial quando a mudança altera sua missão e organização do processo de trabalho - caso da implantação do Sistema Único de Saúde (SUS) que, por força da Constituição de 1988, passou a ofertar de forma descentralizada serviços de saúde a toda população brasileira -, é preciso que ocorram mudanças de atitudes e comportamentos de seus colaboradores. Consequentemente, mudança nas práticas de trabalho, que são a camada mais externa e flexível de uma cultura organizacional (Hofstede, 1980; 1991). 
Esse artigo apresenta os resultados de reflexões feitas pelos protagonistas de um curso de especialização de abrangência nacional, que foi desenvolvido como uma estratégia de "informar e educar" para fomentar a adesão e sustentação dos valores que constituem a identidade do SUS.

Em vinte anos, foi o primeiro curso ofertado com o propósito de conhecer, discutir e disseminar as diretrizes, bases e valores culturais do SUS.

\section{Contexto da intervenção}

O Sistema Único de Saúde (SUS) corresponde a uma política de Estado que estabeleceu forte relação entre democracia e saúde. Esta conexão sistêmica ficou evidenciada a partir da definição em que saúde é o "resultante das condições de alimentação, habitação, educação, renda, meio ambiente, trabalho, transporte, emprego, lazer, liberdade, acesso e posse da terra e acesso a serviços de saúde" (Relatório Final da VIII Conferência Nacional de Saúde, 1986). No espírito dessa lei, o SUS foi criado em 1988, como um sistema único com ações e serviços públicos da saúde organizados em uma rede descentralizada, regionalizada e hierarquizada, de forma a atender às diretrizes da universalização dos serviços, da integralidade da assistência e da participação da comunidade.

A implantação de um sistema avesso às práticas anteriores (centralizadoras na forma de gestão e pouco participativas) representou desafios de ordem técnica, administrativa, política e cultural. É relevante lembrar que desde 1985 havia uma cisão na luta pela reforma da política de saúde: de um lado, os prestadores privados de serviços e, de outro, os atores públicos federais, divididos em dois grandes grupos. O primeiro era formado pelos partidários do comando unificado da saúde. O segundo grupo, pelos defensores da modernização do Instituto Nacional de Assistência Médica da Previdência Social, ligado ao Ministério da Previdência e Assistência Social. Os dois grupos formados por servidores públicos discutiam a necessidade de tornar os serviços prestados mais ágeis, eficazes e descentralizados, mas sob diferentes perspectivas. (BRAsIL, 2006).

O Inamps considerava a descentralização dos serviços prestados e da administração, como forma de adquirir maior agilidade eficácia e eficiência, mas sem abandonar sua posição de centro decisório das políticas de saúde. A proposta defendida pelo Instituto preconizava a modernização da organização previdenciária, sem modificar suas funções de prestador de atendimento médico, de financiador das ações de saúde, de controlador e avaliador dos recursos utilizados, sem considerar a participação social. Quanto à universalização dos serviços prestados, a posição dominante era a de que o atendimento deveria ser restrito à clientela previdenciária e estendido a outros grupos sociais, na medida em que houvesse outros parceiros financeiros e operacionais (BRAsIL, 2006).

Com a aprovação do SUS, evidenciouse a necessidade de um redesenho administrativo do Ministério da Saúde e redistribuição dos servidores, inclusive dos que trabalhavam no antigo Inamps. Para atender as especificidades do SUS, o Decreto no 2.922 de 31, de dezembro de 1998, estabeleceu a estruturação dos núcleos estaduais da saúde (NES). Representantes do Ministério da Saúde nos estados, os núcleos foram formados, majoritariamente, com pessoal oriundo do Inamps. Dessa forma nascia a estrutura para um novo sistema com recursos humanos oriundos de uma organização que, 
institucionalmente, havia se colocado desfavorável ao novo sistema.

No estabelecimento dos NES, não houve um movimento de construção de uma visão compartilhada do objetivo das mudanças que estavam sendo implantadas. Os servidores dos NES foram simplesmente excluídos dos novos processos de trabalho. Inúmeros depoimentos relatam casos de dolo moral que reforçaram a imagem de que os valores e o modus operandi da antiga organização eram superiores aos da nova. O fato de que não houve o reconhecimento da experiência, bem como do conhecimento tácito que detinham, favoreceu o clima de "marasmo" e a cultura da espera pelo tempo de aposentadoria, da inatividade e do conformismo que caracterizam os NES. Concretamente, a formação dos NES foi desacompanhada de um programa institucional de mudança de cultura organizacional e de sistematização dos conhecimentos tácitos adquiridos por experiência ao longo de muitos anos (BRASIL, 1998).

Em paralelo, a ausência de uma definição mais significativa sobre as funções dos núcleos estaduais de saúde fomentou a formação de um ambiente de desconfiança com relação à construção de uma nova cultura organizacional, um processo de inovação e criatividade em que confiança e solicitude são vitais para seu sucesso. De acordo com Choo (2006), o processo de implantação de mudanças em conceitos, crenças e formas de trabalhar esbarra em resistência, particularmente quando é desacompanhado de processo de divulgação e compartilhamento de informações. Processos de mudança criam, em um primeiro momento, desordem no ambiente, e as ações de resistência, antes de qualquer outra intenção, pretendem restabelecer a ordem e retornar à situação de conforto vivida anteriormente.

\section{Antecedentes do curso de especia- lização}

Em 2003, com o propósito explícito de desenvolver um projeto de gestão do conhecimento para identificar, capturar e disseminar o conhecimento no âmbito dos NES, iniciou-se um processo de discussão, promovido pela Coordenação-geral de Recursos Humanos do Ministério da Saúde, sobre a situação dos servidores e trabalhadores lotados nos núcleos. $\mathrm{Na}$ época, o

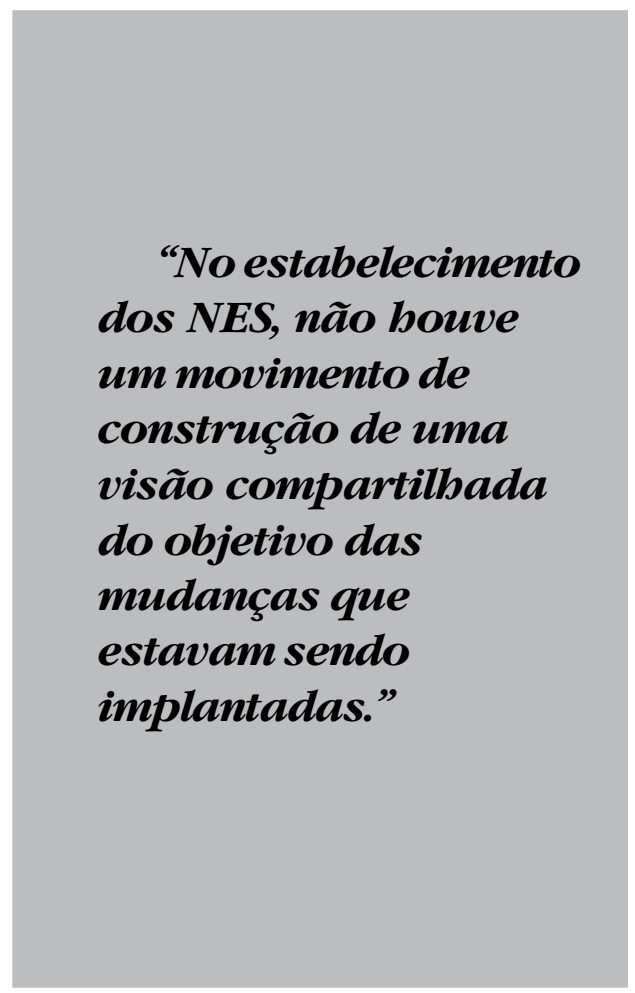

consenso sobre tais trabalhadores era o de que constituíam um grupo desmotivado e que não compartilhavam da missão nem do Ministério da Saúde.

Durante mais de um ano foram feitas reuniões entre representantes do Ministério da Saúde e de instituições de ensino superior dos diferentes estados do Brasil. Ao final do processo foi formada a Rede 
de Parceiros, com vinte e três instituições de ensino superior, que teve como primeiro desafio a formulação de uma proposta de curso de especialização, similar em conteúdo e metodologia, para todos os núcleos estaduais.

Para a elaboração dos objetivos desse curso, foram considerados a missão da instituição, os aspectos históricos e as atuais políticas governamentais, as atitudes e comportamentos dos trabalhadores da saúde, e o compromisso social e político das instituições de ensino superior, em especial, o das universidades públicas.

O objetivo geral do curso proposto foi o de aprofundar o conhecimento da missão e das políticas públicas do Ministério da Saúde, com o propósito de promover uma reflexão sobre a identificação das competências, habilidades e valores capazes de gerar atitudes e comportamentos colaborativos para a superação dos desafios vigentes e futuros da Saúde.

$\mathrm{Na}$ definição do objetivo geral foram considerados os seguintes aspectos:

- Excelência do corpo funcional na administração pública, que deve se traduzir em atendimento mais adequado da razão de ser do órgão público;

- Mudanças organizacionais com participação dos funcionários, para implantação de um sistema que preconiza o atendimento universal, a descentralização e o controle social, entre outros aspectos;

- Problematização como método para favorecer o questionamento, a reflexão e a participação dos servidores no curso; e

- Processos de aprendizagem organizacional que priorizam a atenção às competências para privilegiar o desenvolvimento de atitudes, habilidades e valores.

O grande desafio, naquele momento, era o de promover para todos os servidores o aprofundamento do conhecimento e da compreensão de um sistema de saúde resultante de vários movimentos sociais relacionados à saúde, que congregaram diferentes agentes sociais da sociedade civil, oficializado em 1988 pela Assembleia Constituinte como uma política de Estado, mas que até 2003 não havia sido discutido internamente de modo amplo.

\section{Referencial teórico}

\section{Mudança organizacional}

O processo de gestão do conhecimento, para lidar com as mudanças, inclusive culturais, requer intervenção planejada envolvendo um conjunto de atividades e estratégias para melhorar o funcionamento da organização. Os significados das mudanças com relação à missão, valores culturais, diretrizes e procedimentos operacionais, conforme percebidos por grupos e indivíduos, precisam ser compartilhados e compreendidos para gerar os efeitos desejados. Para que as mudanças possam ocorrer, Bowditch \& Buono (2004) sugerem a promoção da técnica do "descongelamento", que consiste em diminuir a força dos antigos valores, atitudes ou comportamentos. Para tanto, os agentes de mudança podem se valer de reuniões e outras estratégias para questionar os valores atuais e criar condições para que as pessoas enxerguem a necessidade de mudança, o que facilita a implantação de novas ideias e processos.

Para mudar, é preciso ajudar as pessoas a entender a necessidade das inovações organizacionais, mostrar como serão afetadas e o que se espera delas no novo desenho ou na nova missão da organização. Antes que qualquer mudança organizacional possa ocorrer, é preciso preparar a base para a mudança e acompanhar cuidadosamente o processo. Lima e Bressan (2003) 
identificaram cinco dimensões da mudança: a) intencionalidade, b) relevância do impacto da mudança, c) construção social da mudança, d) temporalidade, e e) reposta ao ambiente.

Neiva \& Pantoja (2008) recuperaram o trabalho de Glenn e Malote (2004) em um artigo sobre a complexidade organizacional e a seleção comportamental e cultural, no qual se discute a natureza e os objetivos da mudança organizacional. Glenn e Malote (2004) defendem que as organizações são entidades culturais que estão inseridas em um ambiente complexo e possuem funcionamento e hierarquia também complexos (quanto maior a organização, mais complexa ela é). Essas entidades selecionam comportamentos e padrões culturais para melhor se adaptarem ao ambiente complexo em que estão inseridas. Dessa forma, a mudança organizacional é o gerenciamento da complexidade ambiental e a seleção de contingências comportamentais e culturais para uma melhor adaptação. Conforme o ambiente externo muda, a organização busca mudar as contingências culturais e comportamentais internas para melhor se adaptar.

Numa visão mais recente de mudança organizacional, Paper e Simon (2005) definem mudança como processo contínuo e onipresente no ambiente organizacional. Para eles, a mudança organizacional não pode ser evitada ou ignorada pelas organizações, isto é, ela está arraigada e permeia toda a vida organizacional. Os autores lembram que algumas organizações investem somas significativas em consultoria externa, mas são poucas as que abraçam a transformação e tentam integrá-la em seu planejamento estratégico.

O trabalhador pode perceber a mudança como ameaça ou como oportunidade (Kruglanski; Pierro; Higgins \& CAPOZZA, 2007), como sofrimento e estresse (quando é considerada uma ameaça) ou excitação e motivação (quando percebida como oportunidade). Kuglanski et al (2007) explicam que a mudança (especificamente a episódica) é vista como uma ameaça, pois gera sentimentos de incerteza sobre a natureza e as demandas das novas tarefas e questionamentos sobre as novas habilidades para executá-las, sobre a estabilidade nos seus cargos e sobre a possibilidade de demissão. Os trabalhadores encaram a mudança como um desafio quando ela representa a possibilidade de eliminar rotinas aborrecedoras, permitindo o avanço e a exploração de suas habilidades, e quando gera dinamismo e potencial para o desenvolvimento individual.

$\mathrm{O}$ aspecto de maior interesse para este estudo é a compreensão da dinâmica do fenômeno mudança organizacional, por meio da análise do impacto gerado pela oferta de um curso de especialização focado no entendimento dos princípios filosóficos do Sistema Único de Saúde, nas relações informais da organização.

E para fins desse estudo, partindo-se da literatura mencionada e dos conceitos expostos, mudança organizacional é definida como qualquer alteração na organização que gera impactos, positivos ou negativos, em parte e (ou) em seu todo, e que é percebida e (ou) medida ao longo do tempo. O panorama geral do campo de mudança e de cultura organizacional aqui traçado servirá como base para melhor compreender o contexto no qual o estudo se realizou.

\section{Cultura organizacional}

A alteração de cultura nas organizações é um processo que deve ser tratado com cautela. A cultura é algo tão internalizado, incontestável e invisível para os colaboradores que qualquer tentativa de destruí-la 
ou alterá-la acaba por reforçá-la. Por outro lado, destruir uma cultura antiga e tentar criar uma nova, sem trabalhar adequadamente o passado, acarreta na criação de uma nova cultura, que não se alinhará com as pessoas, processos e redes sociais da organização (ARGYRIS, C. 1999).

A falta de alinhamento entre valores e práticas culturais é percebida e torna doloroso o processo, e tanto a destruição da cultura antiga quanto a criação da nova sofrerão fortes resistências. Por outro lado, se bem compreendido, o processo de mudança pode se tornar motivador para outras alterações necessárias. Quando os membros de uma organização conseguem entender os motivos que levam a uma mudança, a probabilidade de aceitação e identificação com o que a organização se propõe a fazer é maior (ARGYris, C. 1999).

De acordo com Bowditch \& Buono (2004), há basicamente duas maneiras de introduzir mudanças nas organizações. A primeira é fazer com que os integrantes da organização acolham e se comprometam com as ideias, ou seja, envolver as pessoas no projeto e permitir que elas participem ativamente das discussões, inclusive criticando as implicações com as quais não concordem. A outra forma é selecionar e contratar novos colaboradores, dando ênfase às novas crenças e valores. $\mathrm{Na}$ percepção dos autores, por se tratar de um órgão da administração pública, a melhor solução seria a primeira alternativa: educacional.

\section{Programa de educação}

A oferta de programas educacionais para grupos de servidores, em que o objetivo maior seja o fortalecimento institucional, está associada à proposta da reforma gerencial do Estado brasileiro, à Nova Administração Pública, que preconiza, entre outros aspectos, a importância da qualidade de bens e serviços públicos, o que é dependente, essencialmente, das pessoas envolvidas (GONÇALVES, 2006).

Entre as características do novo modelo de administração pública, destacam-se as seguintes: a descentralização administrativa, em que os administradores públicos passaram a ter autoridade e autonomia; a gestão por objetivos e resultados (GEOR), metodologia muito conhecida no ambiente privado, em substituição ao controle rígido pari passo; e a substituição da desconfiança total pela confiança limitada, aspectos que incidem diretamente nos processos de aprimoramento e desenvolvimento de pessoal (GONÇALVES, 2006).

O reconhecimento da necessidade de gestão do conhecimento e do compromisso institucional faz com que os programas educacionais se concentrem em produzir efeitos institucionais. É preciso considerar a perspectiva da interface entre o domínio conceitual, representado pelas instituições acadêmicas, e o campo da aplicabilidade, o espaço de trabalho dos participantes, o lócus interno. Os processos se inserem no conceito de educação corporativa, como um conjunto de práticas sistematizadas oferecidas aos trabalhadores de uma organização como estratégia de gestão do conhecimento: armazenamento e compartilhamento nos níveis individuais e grupais (EBOLI, 2004).

\section{Programas de educação na organização}

Programas de educação nas organizações para além do aperfeiçoamento de competências e habilidades específicas, para a realização do trabalho, também estão associados à alteração substancial de crenças, valores e práticas de trabalho consolidadas. Em se tratando de administração pública, esse processo teve como 
um de seus objetivos o equilíbrio entre a missão da instituição e as políticas de Estado e de governo (Eboli, 2004).

Entre as questões mais frequentes, ao se considerar programas de educação em órgãos públicos, pode-se destacar: como manter servidores interessados e comprometidos com a missão institucional, apesar da alta rotatividade de gestores, o que implica a convivência com diferentes projetos políticos ao longo do tempo? Como fomentar a transferência e o compartilhamento de conhecimentos entre servidores e ocupantes de cargos políticos? Como valorizar o trabalho realizado e o conhecimento dos servidores? A estabilidade pode atuar de forma positiva na construção da memória organizacional e no exercício de práticas inovadoras? Todas essas questões referemse à gestão do conhecimento.

As respostas exigem dos gestores uma nova compreensão da prática de formação permanente de pessoal na administração pública, na qual a estabilidade dos servidores pode gerar conhecimentos tácitos por acúmulo de experiências ao longo do tempo e, também, consolidar rotinas defensivas que impedem novas aprendizagens (ARGYRIS, 1999).

\section{Metodologia}

Neste estudo de natureza eminentemente qualitativa, foi utilizada conjugação de métodos para a coleta e análise dos dados, para captar as impressões e compreender o nível de satisfação dos servidores com o curso de especialização. O curso foi realizado no período 2004-2008, em 21 estados, dois deles com turmas de diferentes NES, e para quatro turmas de Brasília. Apenas os servidores de um estado não participaram do curso de especialização, por ausência de interesse dos servidores.
Caracteriza-se como levantamento tipo censo, uma vez que a amostra de 639 participantes corresponde ao universo de pesquisa, ou seja, todos os servidores dos núcleos estaduais da saúde (NES) que participaram do curso de especialização em Políticas Públicas e Gestão Estratégica na Saúde, realizado em todas as regiões do Brasil, nos 21 cursos oferecidos.

As variáveis do estudo cobriram as seguintes dimensões:

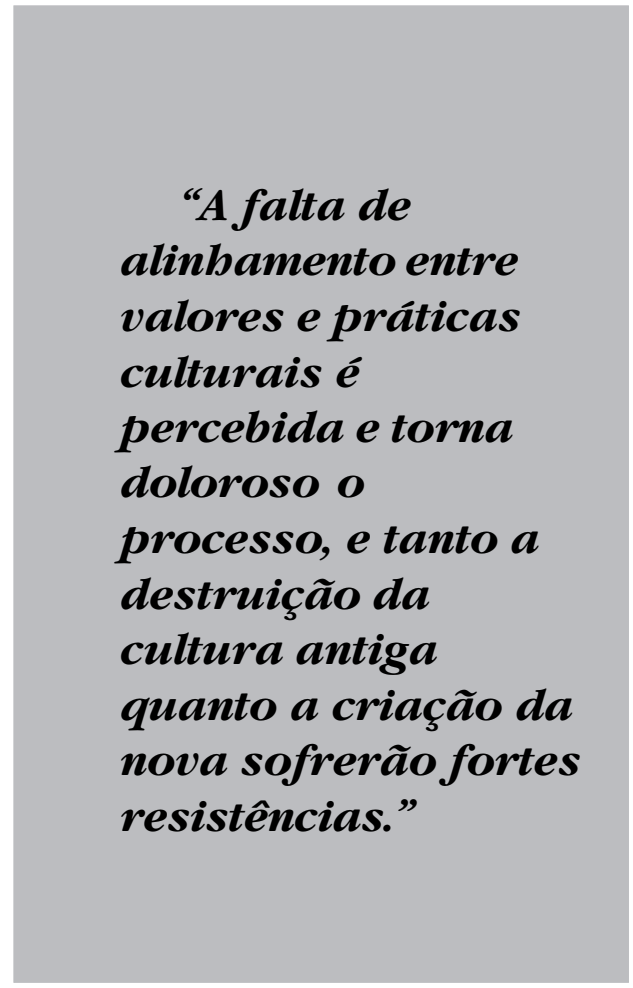

- Contribuição da experiência para mudanças de comportamento;

- Percepção geral das disciplinas: conteúdos, objetivos, bibliografia, carga horária, métodos de aprendizagem e essencialidade para a formação do participante; e

- Relacionamento professor-estudante: acessibilidade fora de sala de aula, autoavaliação de participação no curso e análise da monografia. 
Trata-se, portanto, de uma avaliação do curso realizada pelos alunos, que considerou também efeitos potenciais para o trabalho realizado.

Definidos o conteúdo e a metodologia, a divulgação do curso deveria ser a mais ampla possível, quebrando uma estrutura de comunicação privilegiada e para pequenos grupos. A existência e as condições de participação e seleção foram largamente divulgadas pela rede interna do Ministério da Saúde. Casos pontuais ocorreram em que a informação foi restrita e houve indicação, por superiores hierárquicos, para participação.

Para a inscrição foi exigido dos interessados: a apresentação de uma carta explicitando os motivos pessoais e institucionais, cópia do diploma de graduação e a anuência do gestor. Todos foram entrevistados e tiveram seus documentos analisados por uma banca composta por professores das IES e um representante do Ministério da Saúde. Esse procedimento garantiu transparência e reconhecimento do mérito, o que, por si só, promoveu a valorização dos servidores que se inscreveram e se constituiu em mudança nos processos de seleção para participação em outros cursos.

Como estratégia para coleta, aplicação, tabulação e análise dos dados utilizaram-se:

Entrevista em profundidade, aplicada aos coordenadores um dia antes do início dos cursos. Foi aplicada a 23 diretores de núcleo e 72 coordenadores e professores dos cursos de especialização. As questões da entrevista referem-se ao conteúdo do curso, necessidade de adequação dos módulos às realidades regionais/locais. As respostas foram gravadas e posteriormente transcritas em software de texto. Foi realizada análise temática das respostas dos participantes.

Questionário com 12 questões fechadas, aplicado aos participantes dos cursos de especialização durante o intervalo das aulas; para isso inicialmente eram fornecidas explicações pelo pesquisador para o preenchimento. Para cada questão os alunos deveriam atribuir uma nota de 1 a 10, em que 1 menor nota e 10 maior nota. As questões envolviam o relato das reações dos participantes em relação ao curso, como: percepção geral do curso; entrega dos programas dos cursos; conteúdo; objetivos; bibliografia; alcance dos objetivos; carga horária; método de avaliação do aluno; acessibilidade dos professores fora de sala de aula; autoavaliação da participação no curso; contribuição do curso para mudanças na atuação dentro da instituição; e essencialidade do curso para a formação do aluno como especialista. Os dados foram tabulados mediante a digitação das respostas abertas em software de texto e as respostas foram contabilizadas. Foi realizada análise temática das respostas abertas. Responderam ao questionário 639 participantes.

Os dados foram complementados pela realização de um grupo focal em cada um dos locais em que estava sendo realizado o curso de especialização. Todos os alunos foram convidados, mas o grupo tinha um limite de oito participantes.

Grupos focais com os participantes dos cursos, ao final dos módulos. Os grupos focais eram coordenados pelo pesquisador, e contaram com a participação de 191 servidores. As informações foram coletadas nas 24 turmas do curso de especialização, distribuídas por todo o território nacional. As falas foram gravadas e posteriormente transcritas. No grupo focal foram discutidas as questões: o que motivou a fazer o curso; da experiência como aluno; futuro e outros comentários.

O eixo central das análises empreendidas nesta pesquisa contemplou: a avaliação que 
os servidores/alunos faziam do curso, suas expectativas, suas necessidades de reconhecimento do trabalho e de satisfação pessoal, e a monografia realizada.

\section{Resultados}

Mais de 70\% de servidores que participaram do curso tinham mais de 20 anos de serviço, média na instituição. $\mathrm{O}$ curso foi bem recebido pelos servidores. Os que estavam mais próximos de adquirir o direito a aposentadoria foram os que mais apresentaram críticas à ausência de oferta de cursos de boa qualidade a partir da implantação do SUS. Segundo eles, o curso deveria ter sido ofertado imediatamente após a extinção do Inamps.

Para a maioria absoluta dos participantes, este foi o primeiro curso de 360 horas oferecido pelo Ministério da Saúde, sem custo para os participantes. Entre a população pesquisada, apenas quatro pessoas já haviam feito curso com essa carga horária, pago pelo MS. Dos servidores lotados em Brasilia, 28\% já haviam feito cursos de 360 horas, mas patrocinados com recursos próprios.

Os principais critérios relacionados ao trabalho na organização foram os seguintes:

- Ampliação do conhecimento sobre o SUS para melhorar desempenho profissional (gestão do conhecimento e aprendizagem organizacional);

- Contextualização da atividade exercida no MS; e

- Compreensão das origens das mudanças.

O motivo mais mencionado para participar foi o fato de ocorrer durante o horário de trabalho e gratuitamente, de tratar-se de um curso de boa qualidade e que, além de conferir reconhecimento institucional, satisfazia interesses pessoais e capacitava os participantes para outras atividades na aposentadoria.

Foi constatada uma percepção generalizada de que finalmente, após quase 20 anos, estavam entendendo as mudanças ocorridas, mas que, de acordo com os servidores, foram vividas com muito sofrimento e humilhação. O curso, nesse sentido, foi instrumento de resgate da dignidade do servidor. Aprofundou a consciência da pouca governabilidade dos mesmos e o conhecimento de que o Ministério não é um bloco monolítico, mas sim uma organização composta por vários setores que, muitas vezes, não interagem entre si.

A maioria dos gestores permitiu que os servidores estudassem nas horas de trabalho, usassem a internet para pesquisas e se ausentassem do serviço nos dias de aula. Os gestores que também participaram o consideraram fundamental para o fortalecimento do SUS e dos núcleos. Os gestores que se posicionaram contrariamente eram os que discordavam do SUS e os que não viam esta oportunidade como parte de um plano de carreira.

Gestores concordaram que os conteúdos das políticas públicas deveriam ser oferecidos para todos os funcionários e servidores do MS, mas que o curso deixou a desejar em matérias específicas, como, por exemplo, Planejamento Estratégico. Alguns servidores revelaram maior conhecimento dos conteúdos do que os professores, em especial os envolvidos nos processos de auditoria. Outra crítica foi a de que, muitas vezes, o curso não respondeu a questões práticas, como, por exemplo, "como orientar prefeitos em último ano de mandato a usarem os recursos do SUS".

$\mathrm{Na}$ opinião dos gestores, a monografia teria potencial utilidade para o núcleo apenas se abordasse questões de interesse do trabalho. Em um estado, os gestores 
consideraram o método monográfico revolucionário, tanto para o curso quanto para o trabalho, uma vez que fez com que as pessoas trabalhassem conjuntamente. No início, era uma monografia, mas os temas eram desenvolvidos em grupos.

Todas as IES tinham experiência em cursos semelhantes. Algumas procuraram fazer adaptações para participantes que desenvolveram conhecimentos tácitos, situação diferente dos estudantes regulares das universidades. Os professores reconheceram que os participantes estavam motivados, apesar de reclamações quanto à falta de tempo para estudar e pesquisar, seguidas de sugestões para licença especial do trabalho exclusivamente para estudar. Essa situação exige que novos cursos devam passar por uma avaliação da quantidade do conteúdo versus disponibilidade efetiva para leitura, reflexão e discussão.

Todos os professores afirmaram que os participantes mudaram: ficaram mais integrados; comunicaram-se com colegas de outras áreas; aprenderam a trabalhar em grupo; e tornaram-se tolerantes com as diferenças e mais motivados em relação aos seus trabalhos nos NES.

$\mathrm{Na}$ discussão com todas as IES houve unanimidade quanto ao reconhecimento de que esse tipo de curso deve considerar a relação teoria e prática pela metodologia da problematização.

A avaliação positiva por parte dos colaboradores, gestores e professores envolvidos apontou um conjunto de propostas para novos cursos, revelando uma aprendizagem de conteúdo e de metodologia e uma contribuição à educação corporativa no MS.

Todos os entrevistados demonstraram interesse em mencionar os aspectos positivos e inovadores do curso. Os aspectos mais salientados foram:
- O real conhecimento do SUS e das transformações ocorridas;

- A melhoria da autoestima do servidor;

- Os trabalhos em grupo estimularam a reflexão e o estímulo para participação coletiva;

- Troca de experiências e aprendizagem com outros colegas;

- Sentimento de pertencer ao MS; e

- Conhecimento dos processos de gestão, monitoramento e avaliação.

Com o curso, os servidores passaram a compreender a necessidade do trabalho integrado para o bem da população e deles mesmos, bem como o valor e a eficiência do trabalho coletivo. Como não houve acompanhamento posterior, não se pode afirmar se a percepção ao final do curso se consubstanciou em uma nova prática de trabalho.

Com relação às instituições de ensino superior, foram mencionadas como condições ideais:

- A presença constante do coordenador do curso ou de um professor específico para acompanhar as diferentes disciplinas e auxiliar na integração de conhecimentos de forma coletiva;

- Palestrantes e professores devem ter abertura e experiência prática capazes de problematizar o cotidiano profissional;

- Orientadores suficientes para atender às necessidades de aprendizagem da metodologia científica, com prontidão e abertura;

- Instituição com abertura para compreender faltas institucionais e flexibilidade para desenvolver condições para reposição de conteúdos;

- Metodologia que contemple os tempos diferenciados para compreensão do conteúdo;

- Currículos com enfoque no desenvolvimento de competências na área da 
Gestão, como, por exemplo, em Planejamento Estratégico.

- Foi apontada a falta de sintonia entre o conteúdo discutido pelas IES e as necessidades dos participantes; a parte histórica foi entendida como uma oportunidade para formação de compromisso com a política de Estado de fortalecimento do SUS.

Quanto aos núcleos, ficou evidente que, para o sucesso de uma educação corporativa, é necessária flexibilidade interna para desenvolver uma agenda de atividades profissionais contemplando períodos de encontros presenciais, fóruns, palestras e apresentação das monografias para todos os participantes.

O planejamento em relação à indicação de funcionários, sem implicar acúmulo de atividades para os não participantes, foi considerado vital.

Uma preocupação foi constante: a urgência de estratégias para aproveitamento da formação obtida pelos servidores.

Como conclusão geral, pode-se observar a sensibilização para os valores de um programa de saúde contrários à instituição de origem e uma ressignificação dos programas educacionais oferecidos pela instituição atual, concebidos, no momento da avaliação, como estratégia para melhor cumprimento das atividades profissionais. Os participantes também tiveram a oportunidade de autoavaliação e concluíram que, para melhor aproveitamento, é necessário que exista maior compreensão da razão de ser do programa como atividade para o aprimoramento coletivo; que é preciso mudar a mentalidade e entender que há uma desvinculação do curso enquanto ganho apenas pessoal e com fins para formação de carreira, o que implica compromisso dos alunos em desenvolver trabalhos acadêmicos e profissionais dentro de uma atitude de busca de autonomia e assertividade.

A experiência do programa educacional de abrangência nacional originou uma postura crítica também em relação ao MS, particularmente sobre a função dos núcleos regionais diante da política de fortalecimento do SUS. Levou ao reconhecimento de que é necessário acompanhamento dos desdobramentos do impacto de uma massa crítica dentro dos núcleos regionais, na perspectiva

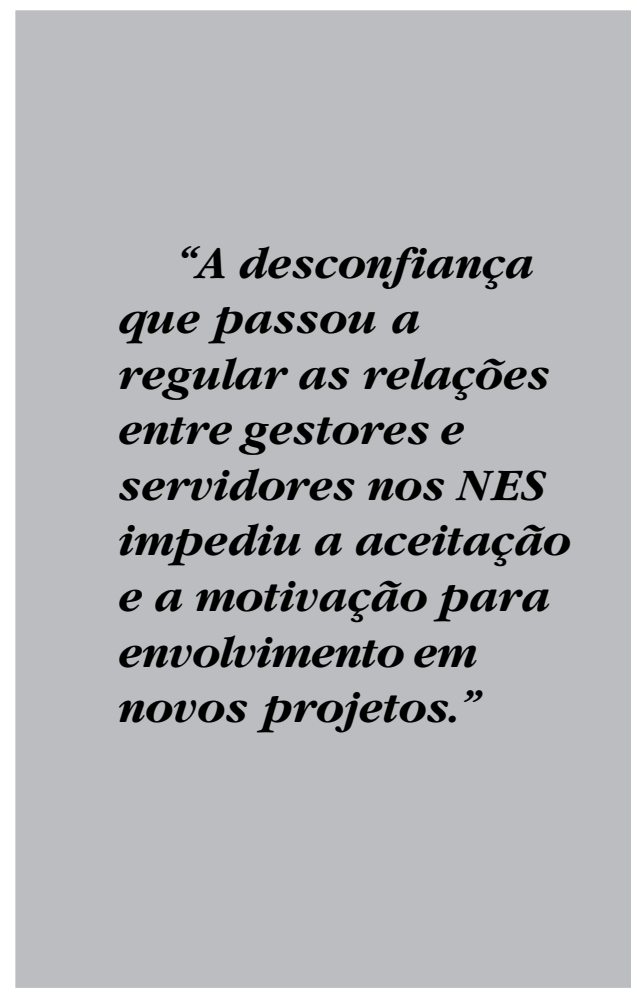

da tolerância para ações novas e inesperadas, oriundas de cargos e funções hierárquicas não superiores.

Foi questionada também a limitação de espaço para uma atuação como agentes participativos e colaboradores nos processos de mudança, necessários para reatualização do papel dos núcleos regionais, uma vez que se percebeu no curso um caráter iniciador e fortalecedor do processo 
de transformação do conhecimento tácito, gerado pela necessidade de atendimento às portarias e normas do SUS.

\section{Considerações finais}

As mudanças ocorridas, quando da estruturação do NES, não foram discutidas com as pessoas envolvidas e as intenções não foram clarificadas, de forma que as alterações causaram confusão, insegurança, desconforto e resistências.

A desconfiança que passou a regular as relações entre gestores e servidores nos NES impediu a aceitação e a motivação para envolvimento em novos projetos.

Entre as rotinas defensivas, o silêncio, o aparente desinteresse e a apatia foram estratégias de sobrevivência que contribuíram para a obstrução do processo de comunicação e, consequentemente, para a aprendizagem organizacional.

O não reconhecimento de competências e habilidades contribuiu para o não envolvimento dos servidores e o insucesso da interiorização dos objetivos do SUS.

Os baixos níveis de satisfação no trabalho e desempenho foram determinantes para experiências frequentes de estresse, estado emocional que tende a ser exacerbado quando se trata de um servidor público, que projeta o vínculo com a empresa para até o momento da aposentadoria.

Para evitar todos esses efeitos nocivos, a permanência de processos de educação, de modo que haja comprometimento dos funcionários com o atendimento dos constantes desafios institucionais, é fundamental. Em paralelo, é preciso criar um ambiente onde prevaleçam o respeito ao indivíduo e a sua capacidade intelectual, onde haja confiança para que os erros possam ser evidenciados e transformados em aprendizado.

(Artigo recebido em janeiro de 2010. Versão final em janeiro 2011.)

\section{Nota}

* As autoras agradecem à Coordenação-Geral de Recursos Humanos do Ministério da Saúde, em especial a Elzira do Espírito Santo, Heloísa Marcolino e Rubio Cesar da Cruz Lima, pela possibilidade de realização deste estudo. 


\section{Referências bibliográficas}

Abrahamson, E. Mudança organiz̧acional. 1ª Ed. São Paulo. 2006.

Argyris, C. On Organizational Learning. 2a Ed. Massachusetts: Blackwell Business, 1999. Bowditch, J. L.; Buono, A. F. Elementos do Comportamento Organizacional. São Paulo: Editora Thomson Learning, 2004.

Brasil. Conselho Nacional de Saúde. Relatório Final da VIII Conferência Nacional de Saúde. 1986. Disponível em: http://conselho.saude.gov.br/biblioteca/Relatorios/ relatorio_8.pdf.

. Constituição Federal 1988. Código Civil. Código de processo civil. Artigo 198. Organizador: Yussef Said Cahali; obra coletiva de autoria da Revista dos Tribunais, com a coordenação de Giselle de Melo Braga Tapai e com a colaboração de Ana Paula Alexandre [et al.]. São Paulo: Revista dos Tribunais, 2005. $7^{\mathrm{a}}$ Ed. rev., atual. e ampl. 1662p. Brasil. Ministério da Saúde. Secretaria de Gestão Estratégica e Participativa. Departamento de Apoio à Gestão Participativa. Coordenação-Geral de Apoio à Gestão Participativa e ao Controle Social. Faleiros, V. P.; Vasconcellos, L. C. F.; Silva, J.F.S.; Silveira, R.M.G. Brasília, 2006.

- Presidência da República. Subchefia para Assuntos Jurídicos. Decreto no 2.922 de 31 de Dezembro de 1998, Artigo 2º que altera o Decreto número 2477, de 28 de Janeiro de 1988, em seu Artigo 2ª, Inciso I, Alínea b, incluindo o Item 5. Disponível em: http://www.planalto.gov.br/ccivil_03/decreto/D2922.htm.

Choo, W. C. A organização do conhecimento. São Paulo: Editora Senac, 2006.

EBoli, Marisa. Educação Corporativa no Brasil: mitos e verdades. São Paulo: Editora Gente, 2004.

Glenn, S. S., \& MALOTT, M. E. Complexity and selection: Implications for organizational change. Behavior and Social Issues, v. 13, n. 2, 89-105, 2004.

Hofstede, G. Culture's consequences: International differences in work-related values. Newbury Park, CA: Sage, 1980.

. Culture and organizations, software of the mind. Intercultural cooperation

and its importance for survival. London: Sage, 1991.

Gonçalves, Sonia M. Goulart. Elementos básicos para a formulação de uma política de gestão do conbecimento para a administração pública federal brasileira. 2006. Dissertação de Mestrado. Universidade Católica de Brasília. Brasília.

Kruglanski, A.; Pierro, A.; Higgins, E., \& Capozza, D. “On the move” or "staying put": Locomotion, need for closure, and reactions to organizational change. Journal of Applied Social Psychology, v. 37, n. 6, p. 1305-1340, 2007.

Lima, S.M., \& Bressan, C. L. Mudança Organizacional: uma introdução. In: Lima, S. M. (org.). Mudança Organizacional: Teoria \& Gestão. São Paulo: Editora FGV, 2003. p. 17-61. 
Neiva, E. R. \& Pantoja, M. J. Redes Sociais e Mudança em um Grupo de Produtores Rurais do Planalto Central. Psicologia, Florianópolis, v. 8, p. 5-24, 2008.

PAes, A. P. Por uma Nova Gestão Pública. Rio de Janeiro: Editora FGV, 2005.

Paper, D., \& Simon, S. Change Research: The Search for a Theoretical Construct. Information Resources Management Journal, v. 18, n. 3, p. 1-1, 2005. 


\section{Resumo-Resumen-Abstract}

Sistema Único de Saúde: a busca do entendimento de seus princípios e sistemas de valores para profissionais do serviço público

Luiza Beth Nunes Alonso, Helga Cristina Hedler e Suely Braga Castilho

No período de 2004 a 2008, o Ministério da Saúde, conveniado com instituições de ensino superior, ofereceu aos servidores dos núcleos estaduais da saúde um curso de especialização sobre Planejamento Estratégico e Políticas Públicas com o objetivo de informar, esclarecer e discutir o Sistema Único de Saúde (SUS). A pesquisa discute esse programa educacional enquanto estratégia de mudança de cultura organizacional no Sistema Único de Saúde. Dois dos principais focos do programa educacional foram a compreensão dos pressupostos filosóficos do SUS pelos funcionários do Ministério da Saúde e a transformação do conhecimento tácito dos servidores em conhecimento sistematizado via elaboração de monografias, na perspectiva da incorporação de uma nova visão sobre o SUS. Foi utilizada abordagem metodológica quali-quantitativa, com uso de questionários, entrevistas e grupos focais com os 636 respondentes que participaram do curso. A análise dos resultados considerou a avaliação que os servidores/alunos faziam do curso, suas expectativas, suas necessidades de reconhecimento do trabalho e de satisfação pessoal, e a monografia realizada. Os resultados indicam a ocorrência de aprendizagem e sensibilização para as mudanças; no entanto, no nível individual fatores organizacionais como a participação, comunicação, reconhecimento de competências e práticas de Recursos Humanos foram mencionados como entraves para o aprendizado e modificação da cultura organizacional. Conclui-se que os processos de aprendizagem desenvolvidos pela organização devem ser processos continuados e não estratégias de ação pontuais.

Palavras-chave: Mudança organizacional; educação no trabalho; cultura organizacional; Sistema Único de Saúde (SUS).

Sistema Único de Salud: la búsqueda del entendimiento de sus principios y sistemas de valores para profesionales del servicio público

Luiza Beth Nunes Alonso, Helga Cristina Hedler y Suely Braga Castilho

En el período 2004 a 2008, el Ministerio de Salud, en virtud de acuerdos con las instituciones de educación superior, ha ofertado a los trabajadores de los centros de salud del Estado un curso de especialización en Planificación Estratégica y Políticas Públicas con el objetivo de informar, aclarar y discutir el Sistema Único de Salud (SUS). Esta investigación analiza este programa educativo como una estrategia de cambiar la cultura organizacional en el Sistema Único de Salud. Dos de los principales focos del programa educativo tuvo como objetivo comprender los supuestos filosóficos del SUS por funcionarios del Ministerio de Salud y la transformación de los conocimientos tácitos de los servidores en un conocimiento sistematizado, lo que ha sido hecho a través de la elaboración de monografías con el fin de incorporar una nueva visión para el SUS. Se utilizó el enfoque metodológico cualitativo y cuantitativo, mediante cuestionarios, entrevistas y grupos focales con 636 participantes que asistieron al curso. El análisis consideró las críticas e sugerencias de los servidores en la condición de estudiantes acerca del curso, sus expectativas, su necesidad de reconocimiento del trabajo y la satisfacción personal, y la realización de la monografía. Los resultados indican la ocurrencia del aprendizaje y el conocimiento de los cambios. Todavía, los factores de organización a nivel individual que inciden en la participación, la comunicación, y en el reconocimiento de habilidades y prácticas del Recursos Humanos fueron citados como barreras para el aprendizaje y para el cambio en la cultura organizacional. Se concluye que los procesos de aprendizaje desarrollados por la organización deben ser procesos de continuidad y no limitarse a estrategias de acción específicas.

Palabras clave: Cambio organizacional; educación en el trabajo; cultura organizacional; Sistema Único de Salud (SUS). 
Unique Sistem of Health: the search for understanding of its principles and value systems for public service professionals

Luiza Beth Nunes Alonso, Helga Hedler and Suely Braga Castilho

In the period of 2004 the 2008, the Brazilian Health Department, in accordance with Institutions of Higher Education, offered to public servers at the State Health Centers a course of specialization on Strategy Planning and Public Politics with the objective to inform, to clarify, to discuss and to understand the Unique Health System (SUS). This research discusses this educational program while as a strategy for culture organization change in the scope of the SUS. Two of the main focus of the educational program had been the understanding of the philosophical assumptions of the SUS by the employees of the Health Department, and the transformation of the tacit knowledge produced by servers along the years in systemized knowledge systemize. This second point was performed through the elaboration of monographs taking in consideration new perspectives about the SUS, and its critical and innovative implementation in their daily routines. Qualitative and quantitative methodology had been applied with the use of questionnaires, focal groups and interviews with the 636 respondents who had participated in the course. The analysis of the results considered the evaluation that the servers, as students, did of the course, its expectations, its necessities of work recognition and personal satisfaction, and the monograph carried on. The results indicate the occurrence of learning and sensitization for changes, however, in the individual level organizational factors such as participation, communication, recognition of practical abilities, and practices of the Human Resources had been mentioned as impediments for the learning and modification of the organizational culture. One concludes that the processes of learning developed by the organization must be a continued process and not only occasional singular action strategies.

Keywords: Organizational change; education in the work; organizational culture, Unique Health System (SUS).

Luiza Beth Nunes Alonso

Mestre e doutora em Administração e Planejamento de Programas Sociais pela Harvard University. Pós-doutorado em Epidemiologia pela Universidade da Califórnia/San Francisco. Diretora, professora e pesquisadora do mestrado em Gestão do Conhecimento e Tecnologia da Informação da Universidade Católica de Brasília (UCB). Contato: lualonso@ucb.br

Suely Braga Castilho

Mestre em GCTI pela UCB. Professora dos cursos de pós-graduação do Instituto Brasileiro de Mercado de Capitais (Ibmec). Contato: suelybcastilho@yahoo.com.br

Helga Cristina Hedler

Mestre em Psicologia e doutora em Psicologia Social, do Trabalho e das Organizações, ambos pela Universidade de Brasília (UnB). Professora e pesquisadora do curso de mestrado em GCTI da UCB. Contato: helga@ucb.br 